\title{
Michalis Nikiforos
}

\section{Uncertainty and Contradiction: An Essay on the Business Cycle}

\author{
June 2015 \\ Working Paper 14/2015 \\ Department of Economics \\ The New School for Social Research
}

The views expressed herein are those of the author(s) and do not necessarily reflect the views of the New School for Social Research. () 2015 by Michalis Nikiforos. All rights reserved. Short sections of text may be quoted without explicit permission provided that full credit is given to the source. 


\title{
Uncertainty and Contradiction:
}

\section{An Essay on the Business Cycle}

\author{
Michalis Nikiforos *
}

June 24,2015

\begin{abstract}
The paper develops a model of economic fluctuations in the medium run and their relation with the short-run macroeconomic equilibrium. The business cycle is the result of two separate forces. On the one hand, there is the Harrodian instability. On the other hand, this instability is contained by the inherent contradictions of capitalism. We focus on two of these contradictions, the profit-squeeze that results from the tightening of the labor market as employment and utilization increase and the financial instability hypothesis, as formulated by Hyman Minsky. With the inclusion of overhead labor, the model can explain the U-shaped behavior of the wage share along the business cycle (wage share decreases for low levels of utilization and increases for higher levels) that prevailed in most of the post-WWII period, as well as the decrease in the wage share as utilization increases that has been observed in the most recent cycles.
\end{abstract}

Keywords: Cycles, Harrod, Oscillations, Distribution, Minsky

JEL Classification Codes: B22, E11, E12, E33

\footnotetext{
*Levy Economics Institute. email address: mnikifor@levy.org. An earlier version of this paper appeared in the Levy Economics Institute Working Paper Collection, WP No. 770. I am grateful to Martha Campbell, Amitava Dutt, Gary Mongiovi, Christian Schoder, Peter Skott and Roberto Veneziani as well as the participants in the 39th Eastern Economic Association Annual Conference in New York, the 16th Conference of the Research Network Macroeconomics and Macroeconomic Policies (FMM) in Berlin and the economic theory workshop at the University of Massachusetts Amherst for useful comments and suggestions. The usual disclaimer applies.
} 


\section{Introduction}

In a recent paper, Nikiforos and Foley (2012) argue that the wage share presents a U-shaped behavior along the business cycle. For low levels of utilization, the wage share decreases as utilization increases and for higher levels of utilization, the wage share increases together with utilization. Figure 1, which is taken from that paper, presents the actual data from the US economy for the period 1947-2010, which confirm — at least prima facie - this kind of behavior for distribution. ${ }^{1}$ Another phenomenon that stands out in the figure is the redistribution of income against wages that has taken place over the last three decades. The relative stationarity of distribution in the first three and a half decades after WWII has been followed by a decline of the wage share since. As a result, there were downward shifts of the U-shaped distributive schedule.

Figure 1 around here

The evidence of a scatter plot, like the one of Figure 1 cannot be conclusive because of the so-called endogeneity problem. Distribution and utilization are determined together through channels where the causality runs both ways. More precisely within the Structuralist (or Kaleckian, or Post-Keynesian) theoretical framework, they are determined through the interaction of the demand and distribution schedules. In the first one, the causality runs from distribution to utilization, while, in the second one it runs the other way around. Nikiforos and Foley (2012) go to great length to try to solve this problems. They use Instrumental Variables within a Two Stages Least Squares framework and verify econometrically the U-shaped behavior of distribution.

This statistical conclusion, taken together with the observations of figure 1 imply a mechanism for the business cycle. The cycle is driven by changes in demand, which interacts with a stable - at least in the medium run-U-shaped distributive schedule. This kind of mechanism is presented in figure 2a. Equilibrium is the result of the interaction of distribution and demand; for instance, when demand is at the level $D 0$, equilibrium is at point $A$. The stable distributive schedule is the base of the cycle, which is driven by shifts of the demand schedule. In the upswing the demand schedule shifts to the right: in our graph shifts from $D 0$ to $D 1$, the new equilibrium in this case is $B$. In the downswing demand shifts to the left, to $D 2$, and equilibrium settles at low utilization levels at point $C$.

\section{Figure 2 around here}

More recent data on distribution and utilization add another interesting dimension to the issue at hand. In figure 3 we present data for the period 2001-2013. It is clear that the recovery after the recession of 2001-

\footnotetext{
${ }^{1}$ For the source and the method of compilation of the data of the figure the reader can refer to the appendix of Nikiforos and Foley (2012).
} 
2002 did not lead to a profit squeeze. Instead, as demand increased and the economy recovered, the wage share decreased and then stabilized in 2007. This is a starkly different picture than the previous figures with the steep upward-sloping distribution at the peak of the cycle. The crisis of 2008 contributed to a further downward shift of the distributive schedule. The recovery of the last five years has been taking place along this lower distributive schedule. Again, as utilization has been increasing, the wage share has been decreasing and thus the distributive schedule is downward sloping. From this point of view the lower-right sub-figure in figure 1 is mistaken. The observations for 2011, 2012 and 2013-which were not available when Nikiforos and Foley (2012) was written — make this interpretation more plausible.

\section{Figure 3 around here}

This observation is important because it shows that the weakening position of the workers vis-à-vis the capitalists during the neoliberal period presents itself not only with the downward shift of the distributive schedule but also with a change of the shape of this schedule; a change in the behavior of distribution along the business cycle. The increase in utilization during a recovery does not lead to a squeeze of the profit share as it used to.

This change does not affect the basic underlying mechanism of the cycle. Again, the stable distributive schedule - which is now downward sloping - is the base of the cycle, which is driven by shifts of the demand schedule, as in Figure 2b. Again, in the upswing the demand schedule shifts to the right from $D 0$ to $D 1$ and in the downswing to the left, to $D 2$.

The purpose - and the contribution - of the present paper is to present a mechanism that explains this behavior of distribution along the business cycle (U-shaped or downward sloping). Towards that goal we develop a model of the business cycle, that combines the canonical structuralist analysis of growth and distribution, with the instability principle, set forth by Roy Harrod and the Marxian insights about the contradictory nature of the capitalist system.

Harrod (1939) is exploring the dynamic dimension of the world described by Keynes three years earlier in The General Theory. Output is determined from the demand side and fundamental uncertainty is prevalent. The uncertainty leads to the falsification of expectations, which (the falsification) then has an impact on demand and the output. As a result the economy is fundamentally unstable and will tend to over-expand or to plunge into recessions. ${ }^{2}$

\footnotetext{
${ }^{2}$ It is probably redundant to say how different is this world from a neoclassical supply-led rational expectations world. If expectations were never disappointed and output is determined on the supply side, the only way to have cycles is with random and inexplicable shocks to (total factor) productivity and thus supply.
} 
On the other hand, the Harrodian instability is contained by the internal contradictions of the capitalist system. To paraphrase the famous aphorism, each stage of the cycle contains the seeds to its own destruction. In this paper we will focus on two of these contradictions. First, the (so-called) profit-squeeze, the increase of the share of the wages for high levels of utilization, which exerts a negative influence on investment and accumulation. In the words of Richard Goodwin $(1967$, p.58) "the improved profitability carries the seed of its own destruction by engendering a too vigorous expansion of output and employment, thus destroying the reserve army of labour and strengthening labour's bargaining power." In other words, the increased profitability will stimulate growth. Higher growth means that employment grows, the reserve army of labor is destroyed and the labor market becomes tighter and that in turn leads to and increase in the wages and decrease in profitability. This decrease in profitability after a certain point will push the economy downwards, the growth will fall, the reserve army of labor will be replenished, the wages will thus fall and the profitability will increase again. Figure 1 confirms this squeeze on the profit share at high level of growth and utilization. ${ }^{3}$

Another "contradiction" which is particularly relevant for understanding the fluctuations of the last two decades is the financial instability hypothesis of Minsky $(1975,1986)$. "In a capitalist environment, stability is destabilizing"(Minsky, 1985, p.12). Protracted periods of growth and increasing utilization will tend to increase the financial instability of the system; the capitalist economies will tend to move from "a financial structure dominated by hedge finance units to a structure in which there is large weights to units engaged in speculative and Ponzi finance" Minsky (1992, p.8). The increased instability will then at a certain point have a negative effect on growth and utilization. ${ }^{4}$

Our modeling approach is novel in the related literature. Usually, the cycles in the growth distribution space are derived by specifying two first order differential equations, one for distribution and another for some component of growth or utilization. Instead, our model is based on a second order differential equation for expectations as a component of investment, demand and growth, while distribution remains static. This approach wants to emphaisize the aforemntioned idea that the cycle is driven by changes in demand, which interacts with a stable distributive schedule (as in figure 2).

A corrolary of this modeling choice is that in our formulation, as opposed to the majority of the related Kaleckian literature (e.g. Barbosa-Filho and Taylor (2006) or more recently Assous and Dutt (2013) and

\footnotetext{
${ }^{3}$ Goodwin formalized an argument which was first formulated by Marx in Chapter 25 of The Capital(1976). A similar behavior of distribution has been proposed among others by Barbosa-Filho and Taylor (2006), Bowles and Boyer (1988), Davidson (1972), Foley (2003), Garegnani (1992), Kurz (1994), Gordon (1995), Taylor (2004), Shapiro and Stiglitz (1984), although there are stark differences in the rationale behind it.

${ }^{4}$ It is important to note that we do not mean that the Marxian contradictions of the capitalist system are limited to negative feedback mechanisms like the profit-squeeze or the financial instability hypothesis, or that all the Marxian contradictions are somehow related to the business cycle.
} 
Kiefer and Rada (2014)), the demand and distribution schedules are not treated as "nullclines." Therefore, the economic fluctuations are not interpreted as the dynamics of the variables around the schedules, but rather as shifts of the schedules themselves. For our present purposes this approach has the advantage that it can provide a coherent and unified framework to explain both the U-shaped and downward sloping behavior of distribution.

Finally, a central role in our argument is played by overhead labor. The combination of overhead labor with a profit-squeeze leads to the observed U-shaped behavior of the wage share. In there is no profitsqueeze, overhead labor creates the downward sloping distributive schedule that we have been observing recently. The importance of overhead labor has been emphasized by many scholars of economic fluctuations like Harrod (1936, ch. II) and Kalecki (1971, ch. 5). A more recent discussion is provided by Assous and Dutt (2013, p. 1411-1412).

The paper proceeds as follows. The next section states a simple stripped-down version of the Kaleckian model in the short run. Section 3 examines the behavior of the system in the long run. Sections 4 and 5 show how the model can explain the U-shaped and the downward sloping behavior of distribution of figures 1 and 3. Section 6 concludes.

\section{The Short Run}

We can now turn to the Structuralist-Kaleckian model of growth and distribution. ${ }^{5}$ We distinguish between the short and the medium run. The crucial difference between them is that in the short run, expectations have been formed and are given. In the medium run the economic actors observe the short run outcomes and modify their expectations. ${ }^{6}$ Note that Harrod himself makes a similar distinction when he talks about dynamic and static equilibrium. He writes that "normally the latter is stable and the former unstable" (1939, p.21). In the context of our discussion static equilibrium resembles what we call short run, while the dynamic equilibrium is our medium run when expectations change and instability prevails.

The setup of the model is standard. As was mentioned in the introduction the economy moves around the demand and distributive schedules. In a closed economy without government sector, demand is determined by the saving behavior of workers and capitalists and the investment behavior of the firms. The income of the economy is distributed between wages and profits.

\footnotetext{
${ }^{5}$ The origins of the the structuralist analysis can be found in the classical political economists, John Maynard Keynes and Michal Kalecki (e.g.1971). In its modern form it has been developed by Steindl (1952), Rowthorn (1981), Taylor (1983, 1990, 2004), Dutt (1984, 1990), Amadeo (1986), Kurz (1990) and Marglin and Bhaduri (1990).

${ }^{6}$ This distinction between different time horizons is common in the literature. See for example Dutt (1997), Lavoie (1995), Skott (2010).
} 
Investment (normalized for capital stock) can be defined as $g^{i}=I(\pi, u)$, where $\pi$ is the profit share, $Y$ and $\bar{Y}$ is output and potential output respectively and finally $u=Y / \bar{Y}$ is capacity utilization with $I_{\pi}>0$ and $I_{u}>0$ (the subscript stands for the partial derivative for this variable). The positive effect of utilization can be justified by the desire of the firm to hold excess capacity to face an unexpected rise in demand (Steindl, 1952). We can also think of it in terms of the acceleration principle. Moreover, as Kalecki and Robinson (1962) pointed out investment depends on expected profitability and firms form their expectations about future profitability based on the current profitability. Higher current profitability also allows the firm to finance a bigger part of its investment through internal funds and eases the access to the capital markets. Current profitability is measured by the profit rate $r=\pi u$; since utilization has already been included as an argument in the investment function it is enough to include the profit share as an additional argument.

For the purposes of the present paper we will assume a simple linear functional form for the investment function

$$
g^{i}=\gamma+\alpha_{1} u-\alpha_{2} \psi
$$

where $\psi=1-\pi$ is the wage share, $\alpha_{1}, \alpha_{2}>0$, and $\gamma$ symbolizes the expectations of the entrepreneurs about the growth rate. ${ }^{7}$ High expectations for the future growth rate boost investment and demand. The opposite happens when the future looks bleak. If someone is willing to put animal spirits in algebraic form, $\gamma$ is a simple way to do it. A spontaneous urge of the entrepreneurs to action rather than inaction, would then be expressed with a high $\gamma$, and would of course increase investment and growth. The opposite would happen in a crisis period like ours when inaction is usually preferred over action. It is important to keep in mind that in the short run $\gamma$ is exogenous.

On the other hand, total saving (normalized for the capital stock) is $g^{s}=S(\pi, u) . S_{u}$ and $S_{\pi}$ are positive. Moreover, we will assume a simple linear form for the saving function

$$
g^{s}=s_{0}+s_{1} u-s_{2} \psi
$$

where $s_{1}$ is the marginal propensity to save out of income and $s_{2}$ the marginal propensity to save out of profits, while $s_{0}$ is a constant.

Equations (1) and (2) define the demand side of the economy. At equilibrium investment is equal to

\footnotetext{
${ }^{7}$ From a formal point of view a correct specification for investment would $g^{i}=\gamma^{\prime}+\alpha_{1}(u-\bar{u})-\alpha_{2}(\psi-\psi(\bar{u}))$, where $\bar{u}$ is a the desired or normal utilization rate, which is constant in the short and medium run. In this case $\gamma^{\prime}$ can be thought of as the growth rate that would prevail when utilization would be equal to its desired level and $\gamma$ in equation (1) is equal to $\gamma^{\prime}-\alpha_{1} \bar{u}-\alpha_{2} \bar{\psi}$
} 
saving, thus

$$
\begin{gathered}
g^{i}=g^{s} \Leftrightarrow \gamma+\alpha_{1} u-\alpha_{2} \psi=s_{0}+s_{1} u-s_{2} \psi \Leftrightarrow \\
\gamma+\left(s_{2}-\alpha_{2}\right) \psi-s_{0}=\left(s_{1}-\alpha_{1}\right) u
\end{gathered}
$$

Given, the so-called Keynesian stability condition $\left(s_{1}-\alpha_{1}>0\right)$, it is easy to see that demand is profit-led $(d u / d \pi>0)$ if and only if $\alpha_{2}-s_{2}>0$; an increase in the profit share increases the utilization rate as long as the propensity to invest out of profits is higher than the propensity to save. The opposite happens when $\alpha_{2}-s_{2}<0$. In this case, demand is wage-led $(d u / d \pi<0)$. In the course of the discussion we will examine the implications of the different demand regimes. Graphically, if demand is profit-led, equation (3) is the downward sloping demand curve in figure 4 (if the economy was wage led, the curve would be upward sloping).

\section{Figure 4 around here}

On the distribution side, the non-linearities will be temporarily ignored until section 4 below. The profit-squeeze can be expressed with a simple linear equation

$$
\psi=\psi_{0}+\beta u
$$

where $\beta>0$ and $\psi_{0} \lessgtr 0$. An increase of utilization will tighten the labor market and will increase the share of wages. Graphically, equation (4) is the upward sloping distribution curve in figure 4.

The short-run equilibrium $A$ will be at the intersection of the two curves. Algebraically, the equilibrium levels of utilization and growth rate can be derived using equations (3) and (4). The short run equilibrium value of utilization is:

$$
u^{*}=u_{0}^{*}+\frac{1}{s_{1}-\alpha_{1}+\left(\alpha_{2}-s_{2}\right) \beta} \gamma
$$

where $u_{0}^{*}=\frac{\left(s_{2}-\alpha_{2}\right) \psi_{0}-s_{0}}{s_{1}-\alpha_{1}+\left(\alpha_{2}-s_{2}\right) \beta}$. The equilibrium rate of the wage share

$$
\psi^{*}=\psi_{0}^{*}+\frac{\beta}{s_{1}-\alpha_{1}+\left(\alpha_{2}-s_{2}\right) \beta} \gamma
$$

where $\psi_{0}^{*}=\psi_{0}+\beta u_{0}^{*}$. Finally, it is also straightforward to find the equilibrium growth rate is:

$$
g^{*}=g_{0}^{*}+\frac{s_{1}-s_{2} \beta}{s_{1}-\alpha_{1}+\left(\alpha_{2}-s_{2}\right) \beta} \gamma
$$


where $g_{0}^{*}=s_{0}+s_{1} u_{0}^{*}-s_{2} \psi_{0}^{*}=s_{0}-s_{2} \psi_{0}+\left(s_{1}-s_{2} \beta\right) u_{0}^{*}$.

One point of interest for our discussion is the relation between the growth rate, the rate of capacity utilization and expectations. From equation (5) we see that utilization always moves in the same direction with expectations as long as the denominator of the fraction is positive. As we discuss in the next section this is tantamount to the system being stable in the short run. Moreover, we will assume that the growth rate moves in the same direction with utilization and expectations. From equation (7) we can see that given the short-run stability of the system, this requires that $s_{1}-s_{2} \beta>0$; that the direct positive effect of utilization on saving, through $s_{1}$, dominates the indirect negative effect because of the profit squeeze, captured with $s_{2} \beta$.

\subsection{The stability of the system in the short run}

The stability of the system requires that - after factoring in the effect of distribution - the reaction of saving to changes in utilization is stronger than the reaction of investment. More formally stability requires that $d g^{s} / d u>d g^{i} / d u$. Using equations (1), (2) and (4) the stability condition is equivalent to $s_{1}-s_{2} \beta>\alpha_{1}-\alpha_{2} \beta$ or $\left(s_{1}-\alpha_{1}\right)+\left(\alpha_{2}-s_{2}\right) \beta>0$.

From the latter inequality, it is clear that the so-called Keynesian stability condition is neither necessary nor sufficient condition for stability. Even if the Keynesian stability condition does not hold the system can be stable as long as $\alpha_{2}-s_{2}>0$ and there is a strong profit squeeze. Similarly the Keynesian stability condition does not guarantee that the system is stable: if $s_{1}-\alpha_{1}>0$ the system is unstable if $\alpha_{2}-s_{2}<0$ and there is a strong profit squeeze.

More precisely, if the Keynesian stability condition holds and demand is profit-led the system is stable, since both $s_{1}-\alpha_{1}>0$ and $\alpha_{2}-s_{2}>0$. If demand is wage-led, the system can be either stable or unstable. It is not hard to see that in the case of wage-led demand the economy is stable if $\frac{s_{1}-\alpha_{1}}{s_{2}-\alpha_{2}}>\beta$, that is, if the slope of the demand schedule is higher than the slope of the distribution schedule. On the other hand, a relatively strong profit squeeze effect makes the system unstable. ${ }^{8}$ In the rest of our discussion-following Harrod - we assume that the short-run equilibrium of the system is stable. ${ }^{9}$

\footnotetext{
${ }^{8}$ When the Keynesian stability condition does not hold, demand is profit-led if $s_{2}-\alpha_{2}>0$ and wage-led if $s_{2}-\alpha_{2}<0$. In the case of profit-led demand the economy can never be stable. When demand is wage-led a relatively strong profit squeeze $\left(\frac{s_{1}-\alpha_{1}}{s_{2}-\alpha_{2}}<\beta\right)$ stabilizes the system, while the system is unstable when the slope of the demand schedule is bigger than the slope of the distributive schedule.

${ }^{9}$ As was mentioned at the beginning of this section (Harrod, 1939, p.21) writes that the short-run, static equilibrium is "usually stable." He continues in the next paragraph: "Some recent writers have been disposed to urge that the static equilibrium is not so stable as is sometimes claimed....I have the impression that this type of criticism exaggerates the importance of this problem, and constitutes to some extent a failure to see the wood for the trees, and that on its own ground the theory of static equilibrium is well able to hold its own."
} 


\section{The Medium Run}

In the medium run economic actors observe the short run outcomes, as described in equations (5) to (6); they contrast them with their previous expectations and form their expectations about the future. In the model this is expressed with the determination of $\gamma$ of the investment function. A possible change of $\gamma$ would affect the short run equilibrium of the next period, which would then lead to a new formation of expectations, and so on and so forth.

This process is usually expressed with the following equation

$$
\dot{\gamma}=\rho\left[\left(g^{*}(\gamma, t)-\gamma(t)\right]\right.
$$

where $\rho>0$ and $t$ stands for time. The dot symbolizes the time derivative, thus $\dot{\gamma}=d \gamma / d t$. If the actual growth rate exceeds the expected growth rate, the expected growth rate increases. Note that if $\partial g^{*} / \partial \gamma>1$ the instability principle prevails. Expectations will never manage to catch up with realized outcomes and the system explodes.

However, economic agents form their expectations based not only on the realized outcome of the last period, but also previous realized outcomes. For example if there is "undue depletion of stock or shortage of equipment" for many consecutive periods before a certain period, say $t$, we would expect this to play a role in the formation of the expectations in period $t$. Harrod was well aware of these lagged effects, although he chooses to ignore them for reasons of economy of exposition. He writes: "the study of these lags is of undoubted importance, but a division of labour in analysis is indispensable, and in this case the neglect is necessary in order to get the clearest possible view of the forces determining the trend and its influence as such"(Harrod, 1939, p.20).

Algebraically, these lagged effects can be expressed by substituting equation (8) with the following one

$$
\dot{\gamma}=\int_{0}^{t} \rho\left[\left(g^{*}(s)-\gamma(s)\right] d s\right.
$$

By time differentiating both sides, equation (9) is transformed into:

$$
\ddot{\gamma}=\rho\left[g^{*}(t)-\gamma(t)\right]
$$

where the double dot stands for the second time derivative. Then, by using the short run equilibrium growth rate from equation (7), equation (10) can be rewritten as 


$$
\ddot{\gamma}+\rho \frac{\alpha_{2} \beta-\alpha_{1}}{s_{1}-\alpha_{1}+\left(\alpha_{2}-s_{2}\right) \beta} \gamma=\rho g_{0}^{*}
$$

This equation describes the behavior of the economy in the medium run. With reference to it and equations (5) to (6), we can infer the behavior of the other variables of the system, utilization, growth rate and distribution.

The important term in equation (11) is the coefficient of $\gamma$, that is $\rho \frac{\alpha_{2} \beta-\alpha_{1}}{s_{1}-\alpha_{1}+\left(\alpha_{2}-s_{2}\right) \beta}$. If this term is negative then $\gamma$ will monotonically diverge from its steady state value. Together with $\gamma$, utilization, the growth rate and wage share will also diverge. On the other hand if the term is positive $\gamma$ will oscillate around its state state value with constant amplitude. We provide a brief discussion of the second order differential equations in the Appendix.

The denominator of this term is positive because of the stability condition, and so is by definition $\rho$. Thus, the question about the sign of the term boils down to the sign of the numerator. It is not hard to see that the numerator is positive if and only if $\alpha_{2} \beta>\alpha_{1}$. In other words, if there is a relatively strong profit squeeze, if investment reacts strongly to changes in distribution or reacts weakly to changes in utilization (or some combination of the three), the term is positive and $\gamma$ presents cyclical fluctuations. An increase in the growth rate will stimulate expectations and will further increase the growth rate. However, as growth and utilization increase profitability is squeezed and investment slows down. This will finally help the expectations to catch up with the realized growth rate. Then the opposite process begins. The growth rate decreases and expectations follow, the labor market loosens and the size of the reserve army of labor increases. This puts pressure on the wage share and slows down the decrease of the growth rate. Again this helps the expectations to catch up with the realized growth rate. This chase of expectations and realized growth continues ad infinitum. ${ }^{10}$

\section{Figure 5 around here}

The second order differential equation (11) can be solved for $\gamma(t)$. The complete solution is

$$
\gamma(t)=\gamma^{s s}+A_{1} \cos (\theta t)+A_{2} \sin (\theta t)
$$

where $\theta=\left[\rho \frac{\alpha_{2} \beta-\alpha_{1}}{s_{1}-\alpha_{1}+\left(\alpha_{2}-s_{2}\right) \beta}\right]^{1 / 2} / 2$ and $\gamma^{s s}=\frac{s-\alpha_{1}+\alpha_{2} \beta}{\alpha_{2} \beta-\alpha_{1}} \rho s-\frac{s\left(\alpha_{2} \psi_{0}+s_{0}\right)}{\alpha_{2} \beta-\alpha_{1}}$. $^{11}$

As we mentioned above, the dynamic behavior of $\gamma$ defines also the behavior of utilization, the growth

\footnotetext{
${ }^{10}$ It is worth saying that if $\alpha_{2} \beta>\alpha_{1}$ and $s_{1}-s_{2} \beta>0$ (which is the condition for $g^{*}$ and $\gamma$ to move in the same direction given the extended stability condition) then also the extended stability condition holds.

${ }^{11}$ Alternatively the solution can be written as $\gamma(t)=\gamma^{s s}+A \cos [\theta t+\phi]$
} 
and the wage share in the medium run. Formally, this can be derived by substituting the solutions of equation (12) into equations (5) to (7). Figure 5 presents the trajectories of the growth rate and the wage share for certain values of the parameters. The oscillations of $\gamma$ drive the growth rate and the wage share, which oscillate with the same frequency. The cycle in this case can be understood as shifts of the demand curve of figure 4 along the stable upward sloping distribution curve.

\section{Non-linear distribution}

The discussion so far has not dealt with non-linearities in distribution. Our system is like the one in figure 4. The discussion of the previous section implies that in the medium run, the changes in $\gamma$ as described by equation (12) will shift the demand curve on the base of a linear distribution curve. How then can this system explain the U-shaped distribution that was discussed in the introduction?

An answer to this can be given by overhead labor. A certain proportion of labor input is necessary for the production, irrespective of the level of economic activity and it does not react to changes in the level of utilization. ${ }^{12}$ The share of the wages of the overhead labor in total income $\left(\psi_{F}\right)$, can be written as

$$
\psi_{F}=\bar{\psi}_{F} / u
$$

where $\bar{\psi}_{F}$ is a constant. In this case $\psi$ is the share of the wages of variable labor in income. Thus the total share of wages in income is

$$
\psi_{T}=\psi+\psi_{F}
$$

The profit squeeze continues to hold, thus the share of variable labor behaves like in equation (4). Equation (14) can be restated as

$$
\psi_{T}=\psi_{0}+\beta u+\bar{\psi}_{F} / u
$$

The total wage share is U-shaped; it decreases as utilization increases for low levels of utilization and increases at higher levels. Under the same specification of the investment function- $g^{i}$ is a function of variable labor share $(\psi)$ and not the total share of labor $\left(\psi_{T}\right)$ - the dynamics of the system are similar with those of figure 2 .

\footnotetext{
${ }^{12}$ Kalecki (1971, ch.6) makes special mention of salaries, which (as opposed to wages) "because of their "overhead" character are likely to fall less during the depression and rise less during the boom".
} 
There are at least three reasons that justify this specification of investment as a function of the variable labor share and not of the total labor share. First, a big part of the overhead labor is the managerial labor, the same people who make the investment decisions for the firm, and therefore do not take into account their share in the wages when they make decisions about investment. Second, a big part of the overhead-fixed labor of the economy is concentrated in the government sector. The fixed wage bill of the government sector is one of the main reasons for the downward sloping segment of the aggregate wage share. However, the investment decisions of the firms in the private sector of the economy are independent of that. Finally, it is worth mentioning that in the most common specification of the Kaleckian model the price is determined with a markup on the average variable cost of production and then investment depends on the gross profit share as defined by this markup. In such a case, even if the markup depends on the average level of the overhead labor - at a lower frequency — and does not vary along the business cycle, our argument remains valid. ${ }^{13}$

\section{Financial Instability}

The profit-squeeze as a result of tight labor market conditions is a thing of the past and maybe of the future, but certainly not of the present. As figure 3 shows the last two recoveries of the US economy have not been accompanied by an increase of the wage share; instead as utilization increases the wage share decreases. This is another demonstration of the weakening position of the workers vis-à-vis the capitalists: not only has the distribution schedule shifted downward but also its shape has changed, and workers cannot claim a higher share of income as utilization and employment increases.

If then there is no profit-squeeze, what contains the Harrodian instability? In this section we discuss how financial instability as introduced by Hyman Minsky $(1975,1986)$ can play this role. The last two downturns of the US economy were caused by the instability in the financial markets and most probably the next one will have the same cause as well.

For reasons of clarity it is important to keep the two kinds of instability - the Harrodian instability and the Minskyan instability - separate. From the point of view of our paper, the Harrodian instability is contained by the Minskyan instability. To paraphrase Minsky, instability is stabilizing.

It is beyond the scope of this paper to make a detailed analysis of Minsky's theory of financial instability. However, we show that even a crude formalization of the financial instability hypothesis, combined with the

\footnotetext{
${ }^{13}$ We must stress that this is not a universally accepted argument in the literature. For example Kalecki (1971, p.51) argues that the increase in the ratio of overhead to variable labor during the slump makes easier a "tacit agreement not to reduce prices in the same proportion as prime costs." Thus, according to Kalecki the markup changes as a result of the changing importance of fixed costs at the business cycle frequency.
} 
framework that we have already introduced in the previous sections can provide some very useful insights to these recent developments.

Important attempts to formalize financial fragility in the context of economic fluctuations include Taylor and O'Connell (1985), Foley (1987), Jarsulic (1989), Skott (1994), Dutt (1995), Keen (1995), Flaschel et al. (1997), Setterfield (2004), Lima and Meirelles (2007), Fazzari et al. (2008), Charles (2008), Ryoo (2010, 2013). This literature formalizes different mechanisms that link the real and the financial sector of the economy and create fluctuations. In many of these contributions a central role is occupied by the interest rate, which has a negative impact on the investment decisions of the firm. On the other hand in good times the system tends to become more and more fragile and the increasing fragility leads to higher interest rates. ${ }^{14}$ A similar argument can be made with credit rationing in the place of interest rate. Another transmission mechanism emphasizes the role of the state of confidence as an argument in the investment function, which erodes as utilization increases. This happens indirectly through the increase of the leverage of the firms, which in turn - at least after a certain point - has a negative impact on the state of the confidence.

Based on the above, we can assume that financial instability $(i)$ has a negative effect on investment. Thus the investment function can be rewritten as

$$
g^{i}=\gamma+\alpha_{1} u-\alpha_{2} \psi-\alpha_{3} i
$$

Moreover, as utilization increases, economic agents tend to move from hedge, to speculative and then Ponzi positions, and financial instability increases together with utilization. We can write this as

$$
i=i_{0}+\delta u
$$

The rest of the specification of the system remains the same as before. Equation (2) describes saving, equation (4) the variable wage share and equations (13) and (15) the fixed and total wage share respectively.

Following the same procedure as before, it is not hard to see that the short run equilibrium value of utilization is:

$$
\bar{u}=\bar{u}_{0}+\frac{1}{s_{1}-\alpha_{1}+\left(\alpha_{2}-s_{2}\right) \beta+\alpha_{3} \delta} \gamma
$$

\footnotetext{
${ }^{14} \mathrm{~A}$ higher interest rate can have a negative impact on investment either directly because it makes borrowing more expensive either indirectly because it increases the interest paid by the firm and thus decreases the internal funds available for investment. In turn, the literature proposes different mechanisms that lead to the increase in the interest rate in the course of the cycle. The interest rate can increase either because demand for loanable funds increases, or because the banks realize the increasing fragility and charge a higher interest rate (or increase their mark-up over the discount rate of the Central Bank) or because they target a constant real interest rate, so as the inflation rate picks up in the upswing so does the nominal interest rate.
} 
where $\bar{u}_{0}=\frac{\left(s_{2}-\alpha_{2}\right) \psi_{0}-s_{0}-\alpha_{3} i_{0}}{s_{1}-\alpha_{1}+\left(\alpha_{2}-s_{2}\right) \beta+\alpha_{3} \delta}$. The equilibrium rate of the wage share

$$
\bar{\psi}=\bar{\psi}_{0}+\frac{\beta}{s_{1}-\alpha_{1}+\left(\alpha_{2}-s_{2}\right) \beta+\alpha_{3}} \gamma
$$

where $\bar{\psi}_{0}=\psi_{0}+\beta \bar{u}_{0}$, and the equilibrium growth rate is:

$$
\bar{g}=\bar{g}_{0}+\frac{s_{1}-s_{2} \beta}{s_{1}-\alpha_{1}+\left(\alpha_{2}-s_{2}\right) \beta+\alpha_{3} \delta} \gamma
$$

where $\bar{g}_{0}=s_{0}+s_{1} \bar{u}_{0}-s_{2} \bar{\psi}_{0}$. The conditions for the economy being wage- or profit-led are the same as before.

Moreover, as before, the stability of the system requires that the reaction of saving to changes in utilization is stronger than the reaction of investment, but in this case we have to factor in not only the effect of distribution but also the effect of financial instability. The extended stability condition can now be written as $s_{1}-\alpha_{1}+\left(\alpha_{2}-s_{2}\right) \beta+\alpha_{3} \delta>0$. As we can see, even if the system is unstable in the $<$ utilization, distribution $>$ dimensions - if $s_{1}-\alpha_{1}+\left(\alpha_{2}-s_{2}\right) \beta<0$ - the Harrodian instability can be contained through the effect of the financial markets as long as $\alpha_{3}$ and $\delta$ are large enough.

In the medium run expectations behave as before. Given the short run equilibrium of the growth rate, the behavior of $\gamma$ is described by the following equations

$$
\ddot{\gamma}+\rho \frac{\alpha_{2} \beta+\alpha_{3} \delta-\alpha_{1}}{s_{1}-\alpha_{1}+\left(\alpha_{2}-s_{2}\right) \beta+\alpha_{3} \delta} \gamma=\rho \bar{g}_{0}
$$

If the extended stability condition holds, $\gamma$ will oscillate around its steady state value with constant amplitude as long as the numerator is positive; if $\alpha_{2} \beta+\alpha_{3} \delta>\alpha_{1}$. In other words, if the combined effect of the profit squeeze and financial instability on investment (through $\alpha_{2} \beta$ and $\alpha_{3} \delta$ respectively) is stronger than the effect of utilization $\left(\alpha_{1}\right)$ the term is positive and $\gamma$ presents cyclical fluctuations.

We can now assume that because of the weak bargaining power of the workers, there is no profit squeeze $(\beta=0)$. The behavior of the system can be seen in figure 6 . The action takes place in the lower panel, the $<$ utilization, instability $>$ space. ${ }^{15}$ As before, the cycle is driven by changes in demand, depicted with shifts of the demand curve. In the upswing demand shifts to the right and the opposite happens in the downswing. The middle panel shows the $<$ utilization, variable wage share $>$ space. Since there is no profit squeeze the variable distributive curve is now horizontal and the variable wage share does not change with

\footnotetext{
${ }^{15}$ Implicitly, we assume that the Keynesian stability condition holds and thus demand is a negative function $i$. This is not a restrictive assumption. Our conclusions would not change much as long as the extended stability condition holds and the numerator of equation (21) is positive (in this case since $b=0$, we need $\alpha_{3} \delta>\alpha_{1}$.
} 
the shifts of the demand curve. Finally, in the upper panel we can see the behavior of the total wage share, the share of variable and overhead labor. For the reasons we explained in the previous sections and since there is no profit squeeze the total distributive curve is downward sloping and thus the wage share decreases as utilization increases during the upswing. This simple model can explain the behavior of the (total) wage share of the last two recoveries as depicted in figure 3.

Three more comments are in order here. First, another interesting case is when there is a profit squeeze (when $\beta>0$ ) but the influence of the profit share on investment is small (for simplicity $\alpha_{2}=0$ ). The economy is obviously wage-led, the variable distributive schedule is upward sloping and the total U-shaped. The important point here is that in this case there is a pseudo-profit-squeeze cycle. If we just limit our attention to utilization and distribution, the cycle appears identical with the cycle of the two dimensional system of the previous sections. This was the case in the US economy in the 1990s. The upswing was accompanied by an increase in the wage share. However, it was not the tightness of the labor market and the high wages that caused the downturn, but rather the bust of the dot-com bubble. If someone limits his attention to the wage-share and utilization the cycle appears almost identical with the profit-squeeze cycles of the 1960s. However, this would be a wrong conclusion.

Second, the variable $i$ can also be thought as a policy variable, like the discount rate of the Central Bank. In this case, equation (17) is a simple version of the Taylor rule; the Central Bank is increasing its interest rate as utilization increases and decreases the interest rate in the downswing when the output gap is increasing. The increase of the interest rate exerts a negative influence of investment and given the condition we have discussed the system behaves in the same way. A mechanism like that, with a central role of the policy decisions of the Central Bank is proposed by Dumenil and Levy (1999).

Finally, an important difference of our formulation with the related literature is that we introduce financial instability at the business cycle frequency. The majority of the above-cited contributions are ambiguous regarding the frequency of the cycle, while Flaschel et al. (1997, ch. 12) and Ryoo (2010, 2013) are explicit that they refer to long waves. Minsky himself had in mind long swings of financial instability, although in some cases there is some ambiguity as well. However, the experience of the last cycles shows that this kind of instability can reemerge sooner and at a higher frequency

\section{Conclusion}

In the previous sections we outlined a model for the business cycle that is built on two main pillars. On the one hand, because of the Harrodian instability, the economy will tend to over-expand or to plunge into 
recessions. On the other hand this instability is contained by the squeeze of the profit share due to the tightening of the labor markets as the utilization and employment increases or by increasing instability in the financial markets.

The mechanism of our model resembles a simple harmonic oscillator from the classical mechanics. To the best of our knowledge it is novel in the related literature. An important difference of our formulation with the related Kaleckian literature is that the demand and distribution schedules are not treated as "nullclines." Therefore, the economic fluctuations are not interpreted as the dynamics of the variables around the schedules, but rather as shifts of the schedules themselves; in our case of the demand schedule.

More importantly the model is able to explain the behavior of utilization and distribution in the post-war period. The first decades after WWI were characterized by a U-shaped behavior of the wage share in the $<$ utilization, wage share $>$ space, while in the two more recent cycles the wage share decreases as utilization increases. Different specifications of our model can explain these patterns.

Finally, although this formulation comes with some strings attached it is a flexible and convenient way to model economic fluctuations at different frequencies. For example, Nikiforos (2014) utilizes this formulation in the examination of long run-fluctuations in growth and distribution. A combination of fluctuations at different frequencies remains a task for the future.

\section{References}

Amadeo, E. J. (1986). The role of capacity utilization in the long period analysis. Political Economy, $2(2): 147-160$.

Assous, M. and Dutt, A. K. (2013). Growth and income distribution with the dynamics of power in labour and goods markets. Cambridge Journal of Economics, 37(6):1407-1430.

Barbosa-Filho, N. H. and Taylor, L. (2006). Distributive and Demand Cycles in the U.S. Economy-A Structuralist Goodwin Model. Metroeconomica, 57(3):389-411.

Bowles, S. and Boyer, R. (1988). Labor Discipline and Aggregate Demand: A Macroeconomic Model. The American Economic Review, 78(2):395-400.

Charles, S. (2008). Corporate debt, variable retention rate and the appearance of financial fragility. Cambridge Journal of Economics, 32(5):781-795.

Davidson, P. (1972). Money and the Real World. MacMillan, London. 
Dumenil, G. and Levy, D. (1999). Being Keynesian in the Short Term and Classical in the Long Term: The Traverse to Classical Long-Term Equilibrium. The Manchester School, 67(6):684-716.

Dutt, A. K. (1984). Stagnation, income distribution and monopoly power. Cambridge Journal of Economics, $8(1): 25-40$.

Dutt, A. K. (1990). Growth, Distribution and Uneven Development. Cambridge University Press, Cambridge, UK.

Dutt, A. K. (1995). Internal finance and monopoly power in capitalist economies: a reformulation of Steindl's growth model. Metroeconomica, 46(1):16-34.

Dutt, A. K. (1997). Equilibrium, Path Dependence and Hystersis in Post-Keynesian Models. In Arestis, P., Palma, G., and Sawyer, M., editors, Capital Controversy, Post-Keynesian Economics and the History of Economic Thought: Essays in Honour of Geoff Harcourt. Routledge, London, UK.

Fazzari, S., Ferri, P., and Greenberg, E. (2008). Cash flow, investment, and Keynes-Minsky cycles. Journal of Economic Behavior \& Organization, 65(3):555-572.

Flaschel, P., Franke, R., and Semmler, W. (1997). Dynamic macroeconomics: Instability, fluctuation, and growth in monetary economies. Mit Press, Cambridge, MA.

Foley, D. K. (1987). Liquidity-profit rate cycles in a capitalist economy. Journal of Economic Behavior $E_{3}$ Organization, 8(3):363-376.

Foley, D. K. (2003). Endogenous Technical Change with externalities in a classical growth model. Journal of Economic Behavior \& Organization, 52(2):167-189.

Garegnani, P. (1992). Some notes on Capital Accumulation. In Halevi, J., Nell, E. J., and Laibman, D., editors, Beyond the Steady State: A Revival of Growth Theory. St. Martin's Press, New York.

Goodwin, R. M. (1967). A Growth Cycle. In Feinstein, C., editor, Socialism, Capitalism and Economic Growth. Cambridge University Press, Cambridge, UK.

Gordon, D. M. (1995). Growth, Distribution, and the rules of the game. In Epstein, G. A. and Gintis, H., editors, Macroeconomic Policy after the Conservative Era. Cambridge University Press, Cambridge, UK.

Harrod, R. (1936). The trade cycle; an essay. The Clarendon press, Oxford.

Harrod, R. (1939). An Essay in Dynamic Theory. Economic Journal, 49(193):14-33. 
Jarsulic, M. (1989). Endogenous Credit and Endogenous Business Cycles. Journal of Post Keynesian Economics, 12(1):35-48.

Kalecki, M. (1971). Selected Essays on the Dynamics of the Capitalist Economy. Cambridge University Press, Cambridge, UK.

Keen, S. (1995). Finance and economic breakdown: modeling Minsky's" financial instability hypothesis". Journal of Post Keynesian Economics, (4):607-635.

Keynes, J. M. (1936). The General Theory of Employment, Interest and Money. Harcourt, Brace \& World, New York.

Kiefer, D. and Rada, C. (2014). Profit maximising goes global: the race to the bottom. Cambridge Journal of Economics. doi: 10.1093/cje/beu040.

Kurz, H. (1990). Technical Change, Growth and Distirbution. In Kurz, H., editor, Capital Distribution and Effective Demand: Studies in the Classical Approach to Economic Theory. Basil Blackwell, Cambridge, MA.

Kurz, H. (1994). Growth and Distribution. Review of Political Economy, 6(4):393-420.

Lavoie, M. (1995). The Kaleckian Model of growth and distribution and its neo-Ricardian and neo-Marxian critiques. Cambridge Journal of Economics, 19(6):789-818.

Lima, G. T. and Meirelles, A. J. (2007). Macrodynamics of debt regimes, financial instability and growth. Cambridge Journal of Economics, 31(4):563-580.

Marglin, S. and Bhaduri, A. (1990). Profit Squeeze and Keynesian Theory. In Marglin, S. and Schor, J., editors, The Golden Age of Capitalism: Reinterpreting the Postwar Experience. Clarendon Press, Oxford.

Marx, K. (1976). Capital: A Critique of Political Economy, Vol. I. Penguin Books, London, England. first publication date: 1867 .

Minsky, H. (1975). John Maynard Keynes. Columbia University Press, New York.

Minsky, H. (1985). The Legacy of Keynes. The Journal of Economic Education, 16(1):5-15.

Minsky, H. (1986). Stabilizing an Unstable Economy. Yale University Press, New Haven, CT.

Minsky, H. (1992). The Financial Instability Hypothesis. The Jerome Levy Economics Institute Working Paper No. 74. 
Nikiforos, M. (2014). Distribution-led Growth in the Long Run. Levy Economics Institute Working Paper No. 814, Levy Economics Institute of Bard College.

Nikiforos, M. and Foley, D. K. (2012). Distribution and Capacity Utilization: Conceptual Issues and Empirical Evidence. Metroeconomica, 63(1):200-229. Special Issue on Kaleckian Growth Theory.

Robinson, J. (1962). Essays in the Theory of Economic Growth. The MacMillan Press, London.

Rowthorn, R. (1981). Demand Real Wages and Economic Growth. Thames Papers in Political Economy.

Ryoo, S. (2010). Long waves and short cycles in a model of endogenous financial fragility. Journal of Economic Behavior \&3 Organization, 74(3):163 - 186.

Ryoo, S. (2013). Bank profitability, leverage and financial instability: a Minsky-Harrod model. Cambridge Journal of Economics. doi: 10.1093/cje/bes078.

Setterfield, M. (2004). Financial fragility, effective demand and the business cycle. Review of Political Economy, 16(2):207-223.

Shapiro, C. and Stiglitz, J. E. (1984). Equilibrium Unemployment as a Worker Discipline Device. The American Economic Review, 74(3):433-444.

Skott, P. (1994). On the modelling of systemic financial fragility. In K., D. A., editor, New Directions in Analytic Political Economy, pages 49-76. Aldershot, Edward Elgar.

Skott, P. (2010). Growth, Instability and Cycles: Harrodian and Kaleckian Models of Accumulation and Income Distribution. In Setterfield, M., editor, Handbook of Alternative Theories of Economic Growth. Edward Elgar, London, UK.

Steindl, J. (1952). Maturity and Stagnation in American Capitalism. Basil Blackwell, Oxford.

Taylor, L. (1983). Structuralist Macroeconomics. Basil Books, New York.

Taylor, L. (1990). Real and Money Wages, Output and Inflation in the Semi-Industrialized World. Economica, 57(227):329-353.

Taylor, L. (2004). Reconstructing Macroeconomics: Structuralist Proposals and Critiques of the Mainstream. Harvard University Press, Cambridge, MA.

Taylor, L. and O'Connell, S. A. (1985). A Minsky crisis. The Quarterly Journal of Economics, 100:871-885. 


\section{Appendix}

\section{A Second Order, Linear, Differential Equations}

A second order, linear, autonomous equation can be written as $\ddot{y}+a_{1} \dot{y}+a_{2} y=b$. The complete solution to this equation is $y(t)=\frac{b}{a_{2}}+A_{1} e^{r_{1} t}+A_{2} e^{r_{2} t}$, where $A_{1}$ and $A_{2}$ are arbitrary constants of integration and $r_{1}, r_{2}=\frac{-a_{1} \pm \sqrt{a_{1}^{2}-4 a_{2}}}{2}$ are the roots of the characteristic equation $r^{2}+a_{1} r+a_{2}=0$. The roots can be either real or complex numbers, depending on the discriminant of the characteristic equation, $\Delta=a_{1}^{2}-4 a_{2}$. If $\Delta \geq 0$ the eigenvalues are real, while if $\Delta<0$ the eigenvalues are complex.

If the eigenvalues are complex, the roots of the characteristic equations can be written as $r_{1}, r_{2}=h \pm \theta i$, where $h=-a_{1} / 2$ and $\theta=\frac{\sqrt{4 a_{2}-a_{1}^{2}}}{2}$. In this case, by using Euler's formula ${ }^{16}$ and some manipulation, the complete solution can be restated as $y(t)=\frac{b}{a_{2}}+e^{h t}\left(A_{3} \cos \theta t+A_{4} \sin \theta t\right)$ where again $A_{3}$ and $A_{4}$ are arbitrary constants of integration.

In the special case that $a_{1}=0$ equation the roots of the characteristic equation are $r_{1}, r_{2}= \pm \sqrt{-a_{2}} / 2$. If $a_{2}<0$ then $r_{1}, r_{2}$ are real numbers and $y(t)$ is unstable. On the other hand if $a_{2}>0$, the discriminant becomes $\Delta=-4 a_{2}<0$. Thus the solutions are complex numbers without a real part (or with a real part equal to zero): $r_{1}, r_{2}= \pm i \sqrt{a_{2}} / 2$. The complete solution is can now be written as $y(t)=\frac{b}{a_{2}}+\left(A_{3} \cos \theta t+\right.$ $\left.A_{4} \sin \theta t\right)$. In this case $y(t)$ will oscillate around its state state value with constant amplitude.

Using some basic trigonometric identities the complete solution can be restated as $y(t)=\frac{b}{a_{2}}+A \cos [\theta t+\phi]$ where $\theta$ is the initial phase angle, the angle for $t=0$, and $A$ is the amplitude of the oscillation. Finally, in both cases $\theta$ is the angular frequency and is equal to $\theta=2 \pi f$, where $f$ is the frequency of the oscillation, the number of cycles per unit of time. Using $f$ we can derive the period $T$, the time required for a complete cycle.

\footnotetext{
${ }^{16}$ Euler's formula states that $e^{i x}=\cos x+i \sin x$ for any real number $x$.
} 


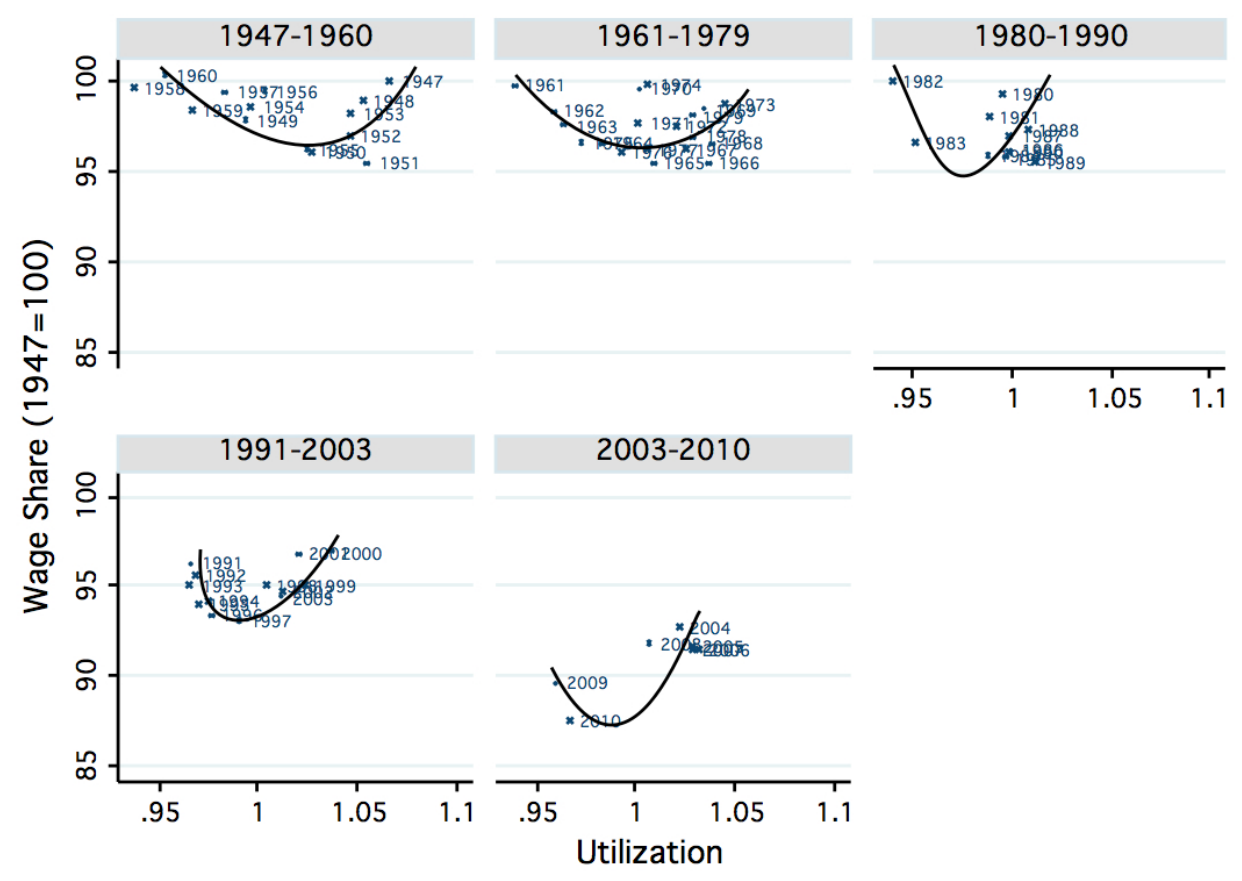

Figure 1: Wage Share and Utilization in the United States, 1947-2010 


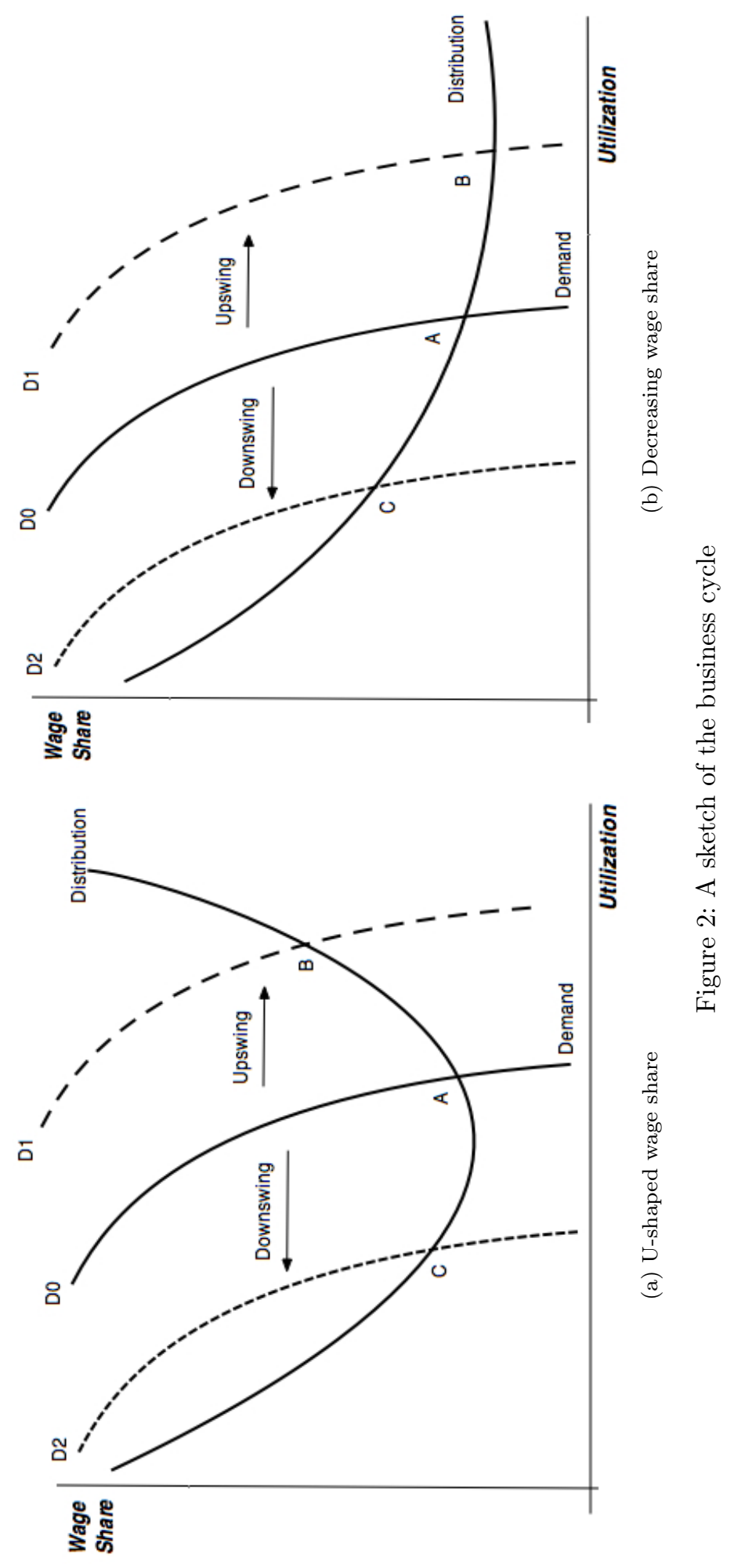




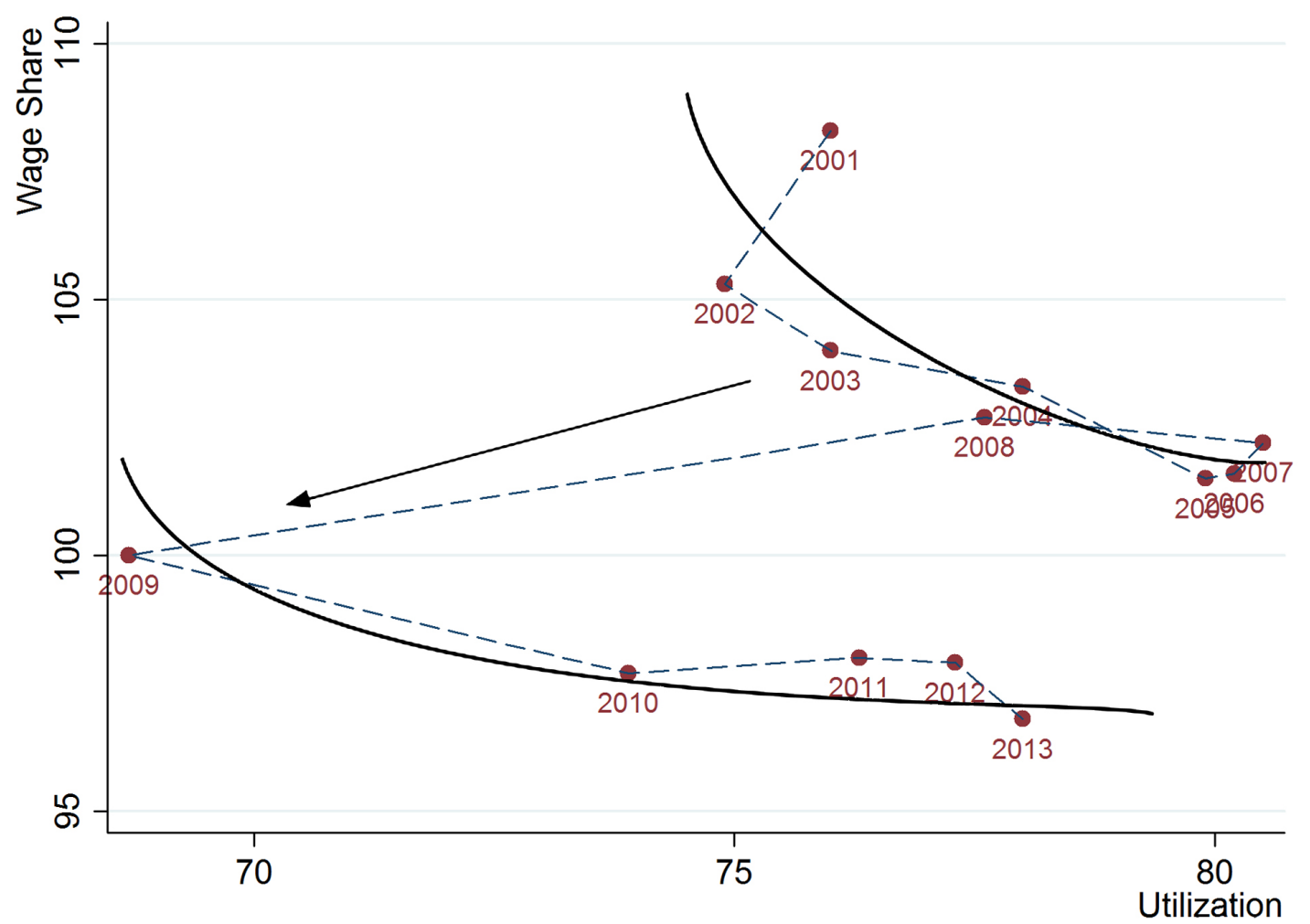

Figure 3: Utilization and the wage share after 2000

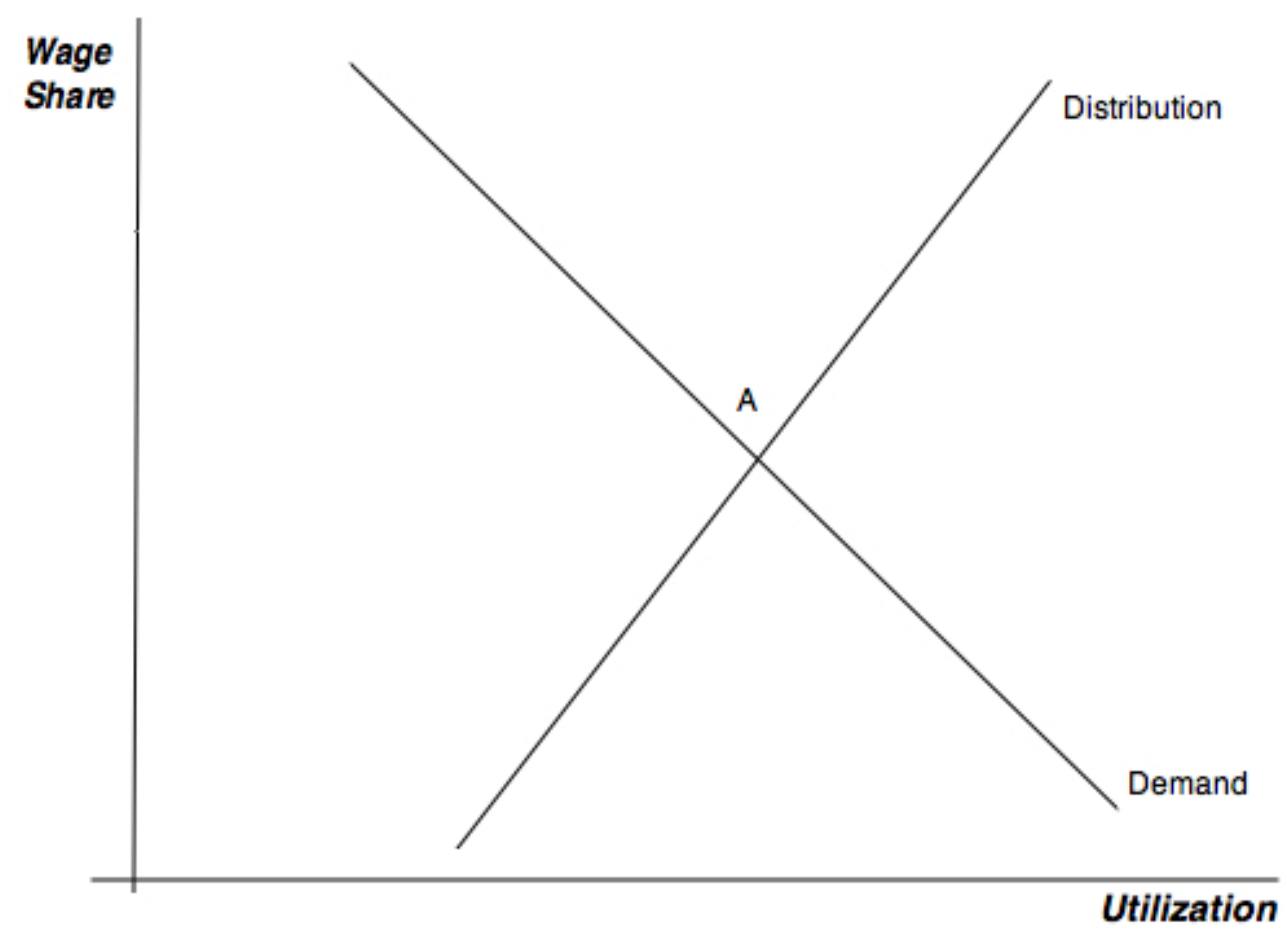

Figure 4: Equilibrium with linear demand and distribution 

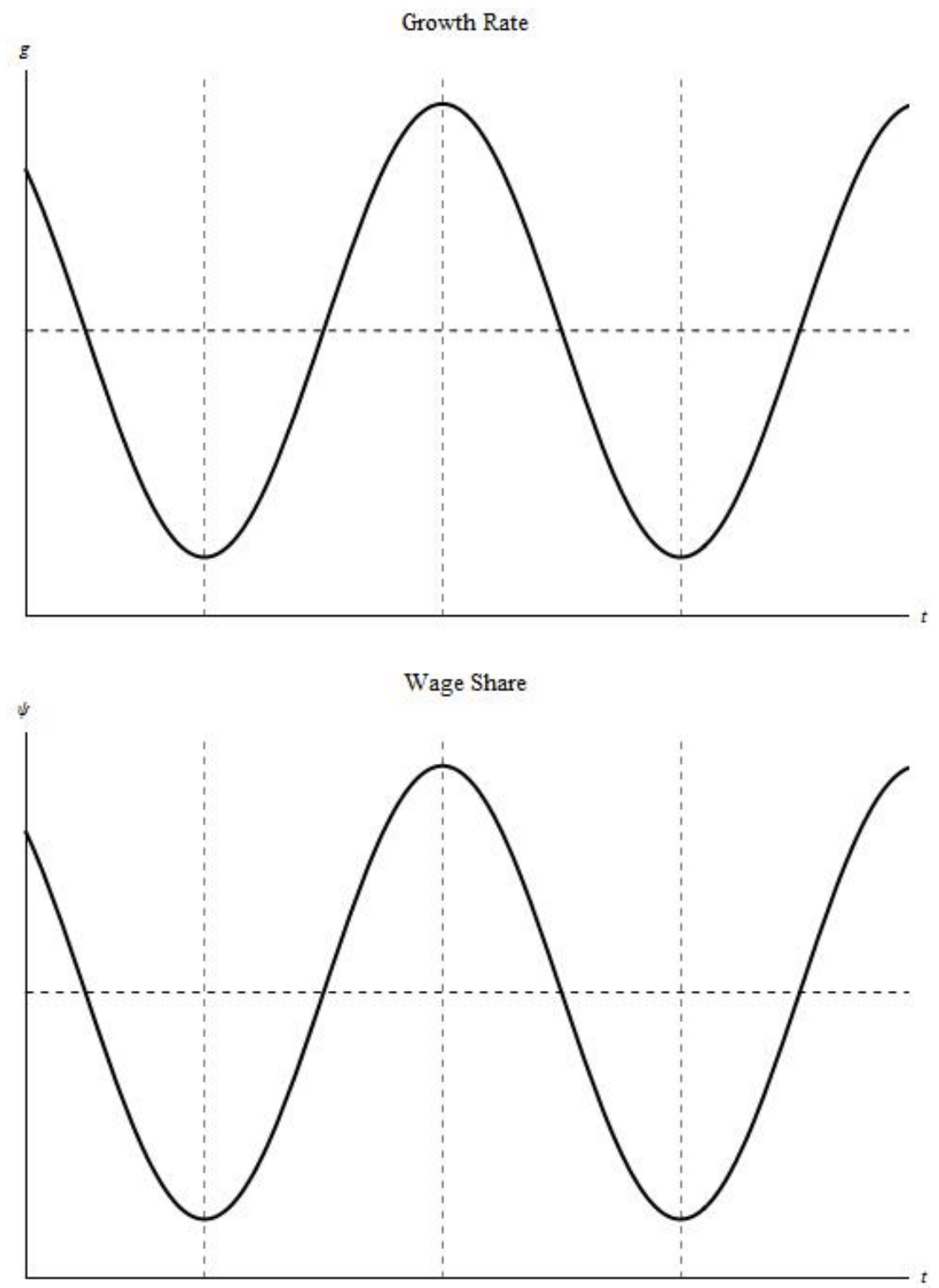

Figure 5: Trajectories of Growth Rate and the Wage Share 


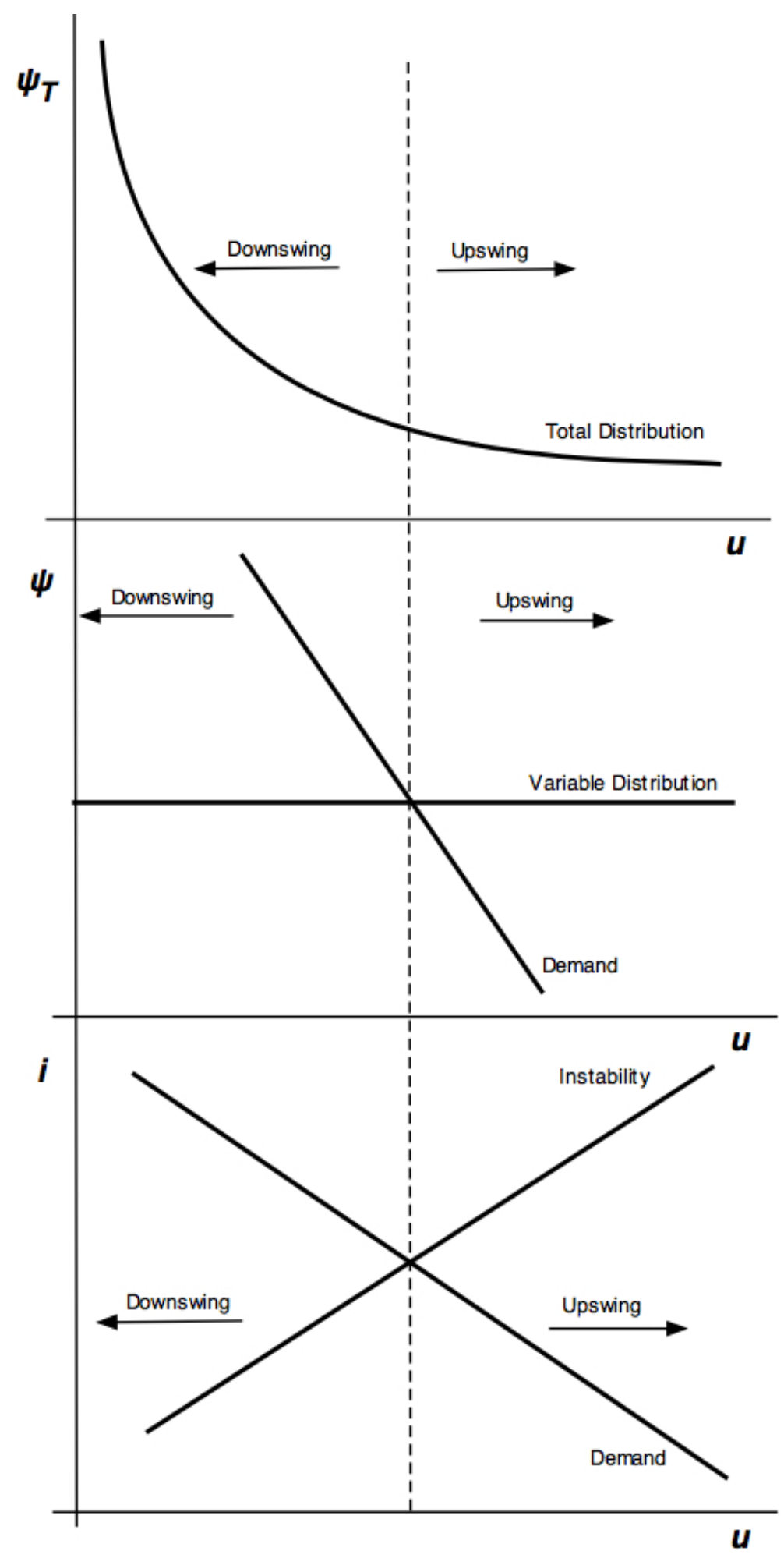

Figure 6: Utilization, Wage Share and Instabilty 